\title{
Temporal summation and reaction time to double-light pulses at suprathreshold levels
}

\author{
TAKEHIRO UENO \\ Osaka City University, Sumiyoshi-ku, Osaka 558, Japan
}

\begin{abstract}
Temporal summation of the visual system was studied at suprathreshold levels by measuring reaction time (RT) to the double pulses that were equal in luminance (L) and duration. The RTs were determined as functions of $\mathrm{L}$ and the stimulus onset asynchrony (SOA) between the onsets of the two pulses. The relation between $\mathrm{RT}$ and $\mathrm{L}$ obtained for a given SOA was found to be a power function with the exponent 1/3. The function relating $L$ to SOA was then derived for each of three criterion RTs. The L-SOA relationship indicated partial summation at SOAs shorter than about $20 \mathrm{msec}$. Inhibition was observed at SOAs longer than about $80 \mathrm{msec}$. These findings were consistent with the predictions from a temporal integration model.
\end{abstract}

The relationship between luminance (L) and duration $(T)$ of a light stimulus can be described by Bloch's law, which states that the product of $L$ and $T$ is constant for a constant visual effect. This law implies that temporal summation of the visual system is complete. Many psychophysical studies, including simple reaction time (RT) experiments as well as threshold ones, have demonstrated that Bloch's law holds within a certain limited range of stimulus duration. The maximum duration at which complete energy summation takes place is termed the critical duration (Hartline, 1934).

A double-light-pulse paradigm has often been employed to examine integration capacity in time. Previous studies have used various response measures, such as detection probability (Bouman \& van den Brink. 1952; van den Brink \& Bouman, 1954), threshold luminance (Davy. 1952; lkeda. 1965), and RT (Grossberg, 1970; Kietzman \& Gillam, 1972). Boynton (1972) summarized the findings of the two-pulse experiments. The magnitude of the critical duration is found to change as a function of pulse duration, retinal location, wavelength, and background luminance (see Table 6 of Boynton, 1972). However, two findings should be noted: one is that under some conditions, the critical duration may be as short as $10 \mathrm{msec}$, and the other is that inhibition occurs when the stimulus onset asynchrony (SOA) between onsets of two pulses is about $50 \mathrm{msec}$.

The purpose of the present study is to examine temporal summation in human vision by using RT as a measure of the visual integral process. The two-pulse paradigm was employed in which both $\mathrm{L}$ and SOA were varied. This is different from the paradigm used by Grossberg (1970). He measured RT to the double-pulse stimulus having a fixed luminance and varying the interval between pulses. The present paradigm made it possible to obtain the L-SOA relationships for several criterion RTs at suprathreshold levels. Regarded from this point of view, the present two-pulse paradigm had a similar nature to Mansfield's (1973) study using the single-pulse paradigm.

In the present experiment, the two stimuli of a double pulse were of equal luminance and equal duration, but were supraliminal. These stimuli were expected to have different effects upon the L-SOA relationship than the near-threshold ones (Grossberg. 1970). The slopes of the function relating $L$ to SOA were less than that predicted from Bloch's law.

\section{METHOD}

\section{Apparatus}

A glow-modulator tube (Sylvania, R1131C), operated at $25 \mathrm{~mA}$, was used as light source. Light from the glow-modulator tube was focused by a lens system onto a small ground-glass plate fastened to the front wall of a box in such a way that it produced a sharp circular target. The target was projected through a Kodak Wratten filter (No. 26) having dominant wavelength of $620 \mathrm{~nm}$ and appeared red, stimulating red cone mechanisms. Therefore, no rod in trusion took place. The luminance level of the target was controlled by calibrated Kodak Wratten neutral density filters. An opaque mask was put on the glass plate to outline the target and to shield it from stray light. The subject could observe the target stimulus through the window, which was cut from the wall opposite to that where the light stimulus was presented. The transilluminated light target was directly in front of the subject and at the level of his eyes. and it subtended a visual angle of $1^{\circ}$. Two small dim red lights $\left(0.15^{\circ}\right.$ in visual angle) served as fixation points, the location of which was symmetrically at $6.5^{\circ}$ on either vertical side of the target. The subject gazed monocularly at the midpoint between the fixation points. so that the target was foveally fixated.

The glow-modulator tube was electronically gated. The waveform and duration of the light pulse, and the SOA between onsets of the pulses were controlled with a transistorized six-channel time regulator (Sanwa, DTR-6), which was driven by a $100-\mathrm{kHz}$ crystal clock and hence accurate to $\pm 0.01 \mathrm{msec}$. When the waveform was displayed by a photodetector on a National VP-546A oscilloscope, it was nearly rectangular, and its rise time and decay time were approximately 30 and $15 \mathrm{usec}$, respectively.

The reaction time from the onset of the first stimulus of a double pulse to the button-pushing response was measured by a TKK digital counter (TW-7010A) and recorded by a TKK digital printer (DP-18). 


\section{Subjects}

Two subjects. A.H. and T.U.. who had corrected normal vision. were used throughout the experiment. Both subjects were trained to an asymptotic $R T$ level in the simple RT experiments.

\section{Stimulus Condition}

Two types of light pulses were used. One was a double pulse. and the other was a single pulse. The two stimuli of the double pulse had equal luminance and equal durations. $2 \mathrm{msec}$. Twenty SOAs. ranging from 4 to $315 \mathrm{msec}$, were investigated. For each of the 20 SOAs. eight luminance levels were employed: $0.2,0.4,1.1,3.5 .10$. 35.110 , and $350 \mathrm{~cd} / \mathrm{m}^{2}$. Therefore, there were 160 combinations 120 SOAs $x 8 \mathrm{Ls}$ ). The lowest luminance was sufficiently intense to yield $100 \%$ detection when presented as a single pulse.

The single pulse had a 2-msec duration and had the same luminance levels as in the double pulse, and thus there were eight combinations ( 1 duration by $8 \mathrm{Ls}$ ). RTs to this single pulse provided a baseline for analyzing the summative interactions of the double pulse.

\section{Procedure}

The subject sat in front of the light-tight box with his head held steady in a headrest. and he was instructed to respond as quickly as possible to each flash by pushing a microswitch with his right fingertip and not to respond on blank trials (trials on which no pulse was presented). Observation of the light stimulus was aluays made with the right eye. After a period of at least $10 \mathrm{~min}$ of dark-adaptation and 20 warm-up trials. the experimental session began.

The subjects served in four sessions. One session consisted of 11 trial blocks-10 SOAs under the double-pulse condition and one under the single-pulse one. Within each of these trial blocks. four of the eight Ls were employed. Therefore. 44 combinations were studied -40 for the double-pulse cond ition and 4 for the single-pulse one. At each of these combinations. 15 stimulus presentations were made and two blank trials were randomly introduced. Under the double-pulse condition. therefore. the subjects responded to the flashes on 600 trials and the blanks on 80 trials. whereas under the single-pulse condition. they observed the flashes on 60 trials and the blanks on 8 trials. The experiment was carried out one session per das.

The experimental sessions for the double pulse were made as follows. The 20 SOAs were divided candomly into IO SOAs each in the first and the second sessions. For each of the SOAs. four Ls were chosen. and 15 RTs to each of the Ls used were measured. In the third and the fourth sessions, the remaining four $L s$ were employed at each of the 10 SOAs, respectively. At the end of the fourth session. therefore, 15RTs were obtained at each of 160 combinations of SOA and $L$. The 'selection of 10 SOAs and $4 \mathrm{Ls}$ and these experimental orders were random for each subject. For the single-pulse condition. on the other hand. four of the $8 \mathrm{Ls}$ were employed in the first session and the other 4 in the second session. Fifteen RTs were measured for each of the Ls. The same procedure was also repeated in the third and the fourth sessions. Consequently, $30 \mathrm{RT}$ s were obtained at each $\mathrm{L}$. The selection of $4 \mathrm{Ls}$ and the experimental order were random.

The light pulses were delivered every $7.8 \mathrm{sec}$ with a foreperiod of r.5 sec between audio warning signal and the first stimulus onset. If the subjects made anticipatory responses. then the sequences were extended until 15 RTs were obtained. A rest period of about $1 \mathrm{~min}$ was taken before a new trial block of pulse presentations.

\section{RESULTS}

As a constant foreperiod was used, the data obtained might be biased by ancitipatory responses. Therefore. the RT values below $130 \mathrm{msec}$ were excluded from data analysis. For subject A.H.. such RTs were 30 and this was $1.14 \%$ of all the observations. whereas for subject T.U.. 36 RTs were found and $1.36 \%$.

The geometric means of RTs to 15 identical double pulses were computed at each luminance level for each SOA. The same calculation was made for RTs to 30 identical single pulses. The lefi-hand part of Figure IA presents an example of the data obtained for subject A.H.. in which the geonetric means of RTs are ploned as a function of log $L$, taking two separate SOAs as a parameter. The curves connecting the dat a points are the function

$$
R T=\frac{k}{L^{n}}+R T_{0}
$$

where $k$ and $n$ are constants and $R T_{0}$ represents the irreducible minimum latency. From Equation 1, we have

$$
\log (\mathrm{RT}-\mathrm{RT} \mathrm{T})=\log \mathrm{k}-\mathrm{n} \log \mathrm{L} .
$$

The reducible latency $R T-R T_{0}$ in Equation 2 refers to the intensity-dependent component. whereas $\mathrm{RT}_{0}$ refers to the fixed motor response component (Manstield. 1973: Piéron, 1952; Vaughan, Costa, \&
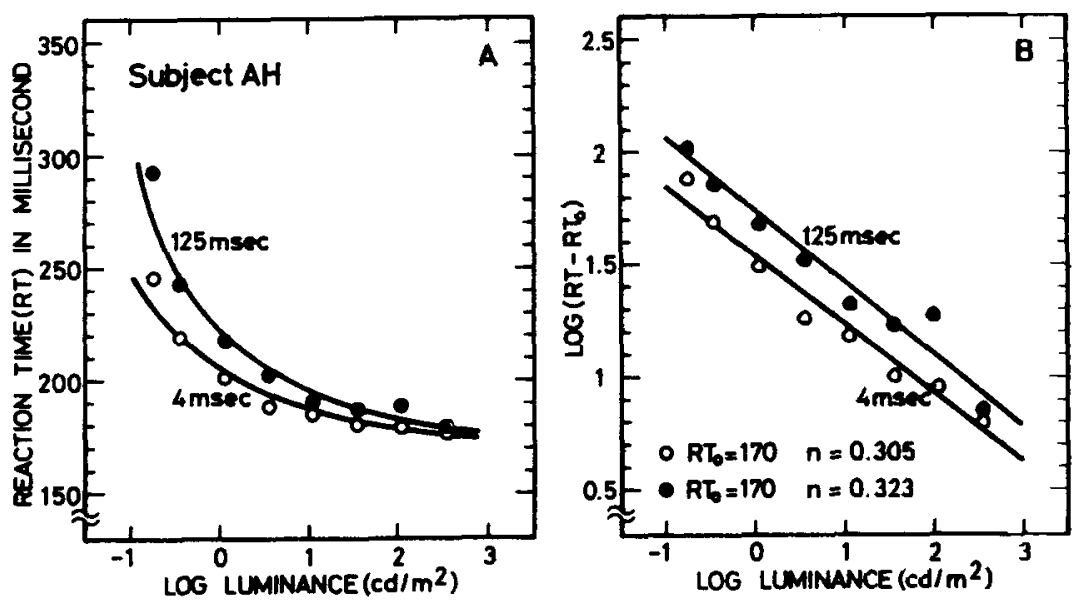

Figure 1. (A) Reaction time as a function of log luminance, with stimulus onset asynchrony (SOA) as a parameter for subject A.H. The curves drawn through the data points represent the RT vs. $\log L$ curve, Equation 1 , fitted by the method of least squares. (B) Log reducible latency (RT-RT ${ }_{0}$ ) as a function of $\log$ luminance. The straight lines connecting the data points represent the theoretical lines by Equation 2. 
Table 1

The Exponent $\mathrm{n}$ and the Asymptotic $\mathrm{RT}_{0}$ (Milliseconds) in Equation 1

\begin{tabular}{ccccc}
\hline $\begin{array}{c}\text { SOA } \\
\text { (msec) }\end{array}$ & \multicolumn{2}{c}{ AH } & \multicolumn{2}{c}{ TU } \\
\cline { 2 - 5 } & $\mathrm{n}$ & $\mathrm{RT}_{\mathbf{0}}$ & $\mathrm{n}$ & $\mathrm{RT}_{\mathbf{0}}$ \\
\hline 4 & .31 & 170 & .35 & 174 \\
5 & .38 & 175 & .38 & 185 \\
6 & .32 & 170 & .29 & 181 \\
8 & .34 & 170 & .30 & 184 \\
10 & .37 & 175 & .36 & 185 \\
13 & .31 & 164 & .30 & 180 \\
16 & .27 & 166 & .33 & 180 \\
20 & .33 & 170 & .35 & 190 \\
25 & .36 & 175 & .39 & 192 \\
30 & .36 & 173 & .30 & 185 \\
40 & .32 & 161 & .34 & 190 \\
50 & .29 & 170 & .33 & 180 \\
65 & .38 & 168 & .43 & 189 \\
80 & .39 & 167 & .41 & 190 \\
100 & .41 & 173 & .33 & 190 \\
125 & .32 & 170 & .29 & 180 \\
160 & .32 & 167 & .25 & 186 \\
200 & .35 & 170 & .27 & 178 \\
250 & .35 & 172 & .35 & 193 \\
315 & .35 & 172 & .37 & 188 \\
Single Pulses & .34 & 168 & .36 & 190 \\
\hline
\end{tabular}

Gilden, 1966). In $\log$ - $\log$ coordinates. Equation 2 expresses a straight line whose slope is the exponent $\mathbf{n}$. Part B of Figure 1 is an example of this.

Equation 2 was fitted to all of the data obtained for the two subjects. The values of $\mathrm{RT}_{0}$ were graphically estimated from the asymptotes of the RT vs. $\log \mathrm{L}$ curves such as show'n in Part $A$. The values of $k$ and $n$ were then estimated by the method of least squares. Table 1 shows the calculated values of $n$ and $R T_{0}$ for the single and double pulses. For subject A.H., the averages of $n$ and $R T_{0}$ are 0.34 and $169 \mathrm{msec}$, whereas for subject T.U., 0.35 and $188 \mathrm{msec}$. No systematic change is tound in the $n$ and $\mathrm{RT}_{0}$ as SOA increases.

To examine to what extent the double pulses interact at suprathreshold levels, the luminance required to produce a criterion value of the quantity $\mathrm{RT}-\mathrm{RT}_{0}$ was calculated by using Equation 2 fitted to the $\log \left(R T-R T_{0}\right.$ ) vs. $\log L$ curves. Figures 2 and 3 show the luminances that are necessary to give three

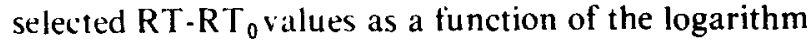
of SOA. The data points for the single pulse also show the luminances to produce the same RT-RT, values as in the double pulse, and their vertical bars present \pm 1 standard error of estimates. The solid horizontal lines in each tigure correspond, respectively, to the luninances calculated for single pulse. Likewise, the dashed lines agree with the vertical bars.

The data plotted in Figures 2 and 3 scatter considerably. However. a general trend may be found. At very short SOAs, the luminances are clearly lower than those for the single pulse, and they increase with the increase of SOA and reach the dashed lines at the SOAs trom about 20 to $30 \mathrm{msec}$. This finding implies that sunmation occurs within this range. At SOAs longer than about $80 \mathrm{msec}$, the values of luminance shift above the regions of the dashed lines, suggesting an inhibitory phenumenon. When SOA becomes more than about $200 \mathrm{msec}$. the luminances tend to return the same levels as the dashed lines. This tendency is found clearly for subject A.H.. but is not so for T.U.

The straight lines fitted at brief SOA parts in Figures 2 and 3 are the function

$$
\mathrm{Lt}^{-\mathrm{m}}=\mathrm{C} \text {. }
$$

"here t represents SOA, $\mathrm{C}$ and $\mathrm{m}$ are constants and $m>0$. Equation 3 could be regarded as a version of Piéron's law, applied to the double pulse. When $\mathrm{m}=1$. Equation 3 implies complete summation. When $0<m<1$, it means partial summation. In the caise of $m=0$. zero summation is obtained, indicating that luminance is independent of SOA. The $n$ values were estinated by using the method of leatst squares. When the criterion $R T-R_{0}$ value becomes 40,70 , and 100 msec, the corresponding $\mathrm{m}$ values for subject A.H. are 0.90 .0 .82 , and 0.75 , respectively, whereas those for subject T.U. are 0.76 . 0.75 , and 0.66 . respectively. Summation tends to be more partial as the RT-RTo value increases.

The values of SOA at which the straight line by Equation 3 intersects with the solid horizontal line were determined. The computed SOA values for subject A.H. are 17.1, 19.7, $22.1 \mathrm{msec}$, respectively, varying the $R T-R T_{0}$ value from 40 to $100 \mathrm{msec}$. The values for subject T.U. are 22.3 .24 .7 , and $34.0 \mathrm{msec}$, respectively. The points of intersection seem to increase with the increasing RT-RT 0 value.

\section{DISCUSSION}

\section{RT vs. L Curve}

The previous RT studies have indicated that the exponent $\mathrm{n}$ in Equation 1 is approximately $1 / 3$ in the single-pulse experiment (Mansfield. 1973; Vaughan

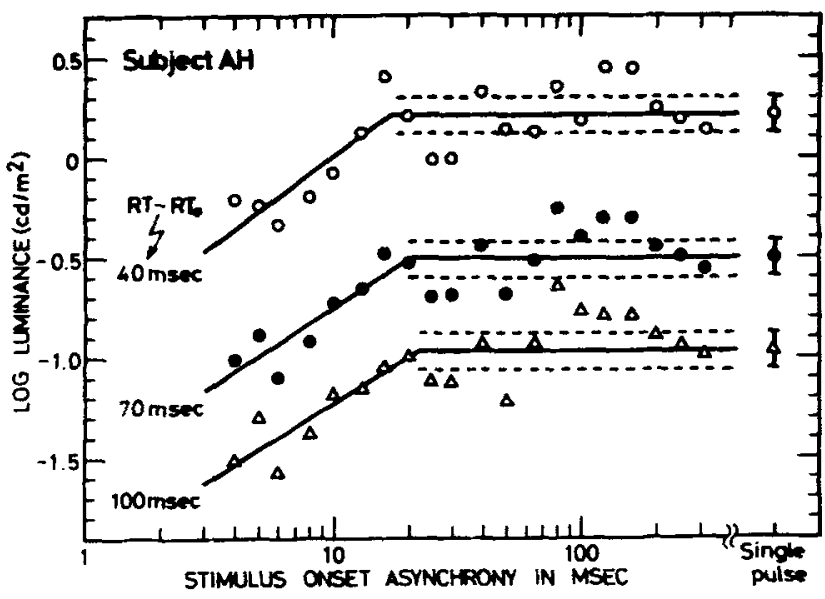

Figure 2. Luminance as a function of SOA, taking the RT-RT, value as a parameter for subject $A . H$. The vertical bars in the single pulse represent \pm 1 standard error of estimates. 


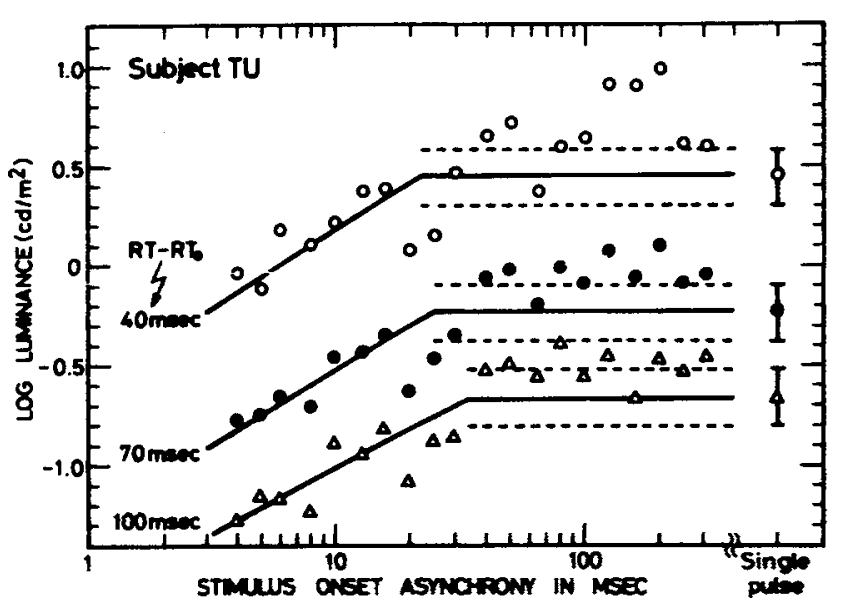

Figure 3. Luminance as a function of SOA, taking the RT-RT, value as a parameter for subject T.U. The vertical bars in the single pulse represent \pm 1 standard error of estimates.

et al.. 1966). Furthermore. Mansfield (1973) found $1 / 2$ for a point source. The $n$ values obtained in the present study are distributed around $1 / 3$, as shown in Table 1. It is to be noted that the exponents under the present double-pulse paradigm become equal to those found in the single-pulse studies. If, however, the two stimuli of a double pulse have equal durations but have unequal luminances, then the same result could not always be expected, particularly when the luminance of the first stimulus is lower within a certain range of SOA.

The minimum irreducible latency $R_{0}$ was expected to keep a fixed value throughout every condition. However. Table 1 indicates that the estimated values of $\mathrm{RT}_{0}$ are distributed within about $20 \mathrm{msec}$ at the center of the means. This variability may be somewhat large. Therefore. the $\mathrm{RT}_{0}$ might be treated as a random variable.

\section{Summation}

The duration at which departure from complete energy summation occurs is termed the critical duration (Hartline, 1934). The critical duration in Grossberg's (1970) RT study is estimated to be $10 \mathrm{msec}$ (see Table 6 of Boynton, 1972). Kietzman and Gillam (1972) found that the critical duration is $11 \mathrm{msec}$ by measuring RTs to the equal-energy luminance- or duration-varied pulses. Of importance is the finding that the critical duration for RT is shorter than the critical durations for such psychophysical measures as response frequency and signal detectability (Bruder \& Kietzman, 1973). Bruder and Kietzman (1973) and Hood and Grover (1974) make explanations for this. ${ }^{1}$

The prior threshold experiments have demonstrated that the critical duration is followed by a period of partial summation that continues up to at least $2 \mathrm{sec}$ (Connors, 1970; Sperling \& Jolliffe, 1965). A similar tendency is also found in the RT studies.
Bruder and Kietzman (1973) found that partial summation for threshold-level stimuli lasts up to $65-95 \mathrm{msec}$ after stimulus onset. As they pointed out, evidence of partial summation for RT can already be seen in the earlier RT studies at threshold (Grossberg, 1970) and suprathreshold (Kietzman \& Gillam, 1972) levels.

In the present study, summation occurs at very short SOAs, as is clear from Figures 2 and 3. However, the summation observed is partial, since the estimated $m$ values in Equation 3 are less than 1 . The average SOAs of intersection are $19.6 \mathrm{msec}$ for subject A.H. and $27.6 \mathrm{msec}$ for subject T.U. These tindings are obviously different from the results found by Kietzman and Gillam (1972). The difference between the present data and their data is probably due to the difference in stimulus manipulations employed. The two-pulse condition of their Experiment II is closely related to the present experimental paradigm. Kietzman and Gillam measured RTs to the double pulse with a fixed luminance, varying the interval between pulses, whereas in the present experiment both luminance and SOA were varied.

\section{Inhibition}

By using a double-pulse increment threshold technique, Ikeda (1965) and Uetsuki and Ikeda (1970) studied the summative power of the human visual system as a function of SOA. Their results indicate that when SOA is very short, the two stimuli of a double pulse summate linearly in the visual system, implying complete energy summation. When SOA is very long, the energies of the two stimuli do not summate, and therefore the threshold that corresponds to the so-called probability summation is obtained. At an intermediate SOA, which is longer than the SOA for complete summation but shorter than that for probability summation. Ikeda and Uetsuki found that the threshold for a double pulse was almost the same as that of a single pulse, implying inhibition.

Ikeda (1965) found such an inhibition at a SOA of 50 or $70 \mathrm{msec}$, depending upon the adapting levels. For relatively low adapting levels, including dark adaptation, the results by Uetsuki and Ikeda (1970) did not show strong inhibition, but rather weak and long-continued inhibition, ranging from about 100 through 300 sec.

In the present $R T$ study, a similar phenomenon can also be found. At SOAs longer than about $80 \mathrm{msec}$, the double pulse brought a longer RT than the single pulse did. Consequently, stronger luminance was required for the double pulse than for the single pulse to produce a certain $\mathrm{RT}-\mathrm{RT}_{0}$ value. This finding appears to be a manifestation of the inhibitory interaction that is originated from the two stimuli of a double pulse. Grossberg (1970) also found that when 
SOA is longer than about $50 \mathrm{msec}$, the RT to a double pulse continues to increase to a peak that is longer than the RT to a single pulse.

At SOAs longer than about 200 msec. the double pulse yielded RTs that fell within the dashed lines for single pulse. This finding also agrees with Grossberg 's (1970) results. As the luminance level used in the present work was well above threshold, this result could be interpreted as follows: When SOA is very long. the two stimuli of a double pulse do not interact in the visual system, and hence the subjects can respond to the first pulse alone.

\section{A Model for Temporal Integration}

The present data might be explained by a conceptual model in which the visual system integrates sensory information from the light pulse. The model proposed here rests on the following assumptions:

(1) The visual system elicits at some levels a response caused by each of the two stimuli of a double pulse. The response presents the bivalent response function that is temporally spread.

(2) At one level of analysis. the system linearly superposes these response functions with appropriate weighting coefficients. The magnitude of the weighting coefticients largely depends on the SOA between the onsets of two pulses.

(3) In order to detect a light pulse. the system continues 10 integrate from the onset of the pulse to a certain time, $\tau_{0}$. along the response function until the value of the integral becomes equal to a fixed criterion value. $k$. The time. $\tau_{0}$ may therefore be seen as a latency time that is consumed in the so-called sensory-decision stage.

(4) The observed RT is composed of a sum of $T_{0}$ and $\tau$. The time $\tau$ represents a delayed latency time in the residual stages, and it is independent of stimulus intensity, pulse duration, and SOA.

Consider first a single-pulse case with moderate luminances. The upper part of Figure $4 \mathrm{~A}$ presents a hypothetical response function. $f(t)$. that originates from a single pulse. The response function is composed of positive (excitatory) and negative (inhibitory) parts. The ordinate is arbitrary. The level at which the response occurs and the levels at which these responses interact are not specified. The exact form of $f(t)$ is difficult to specify. ${ }^{2}$ Considering the findings in the studies quoted belou: however, it seems reasonable to assume that when luminance is not so low, there is a transition at about 70 mosec from the positive part to the negative. The idea that the response function is bivalent is not new. Such an impulse response function has been suggested previously (Kelly, 1961: Ikeda, 1965: Sperling \& Sondhi, 1968: Uetsuki \& Ikeda. 1970). Part A indicates that the visual system detects the stimulus at time $\tau_{0}$ by Assumption 3. The RT is then observed as $\tau_{0}+\tau$. based upon Assumption 4 .

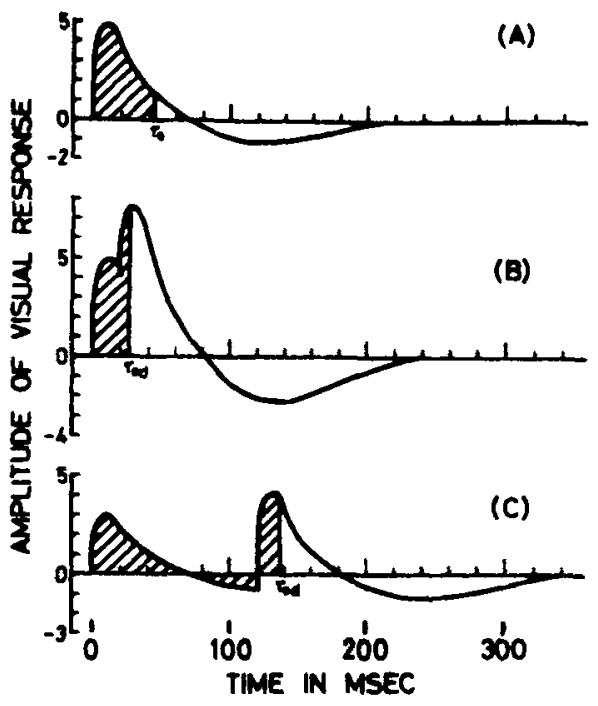

Figure 4. (A) A hypothetical response function that ordinate from a aingle-light pulse. The visual arstem detects the light pube at time $\tau_{0}$. (B) Summation by cuperposition of the hypothetical responce functions due to the two stimull of a double pabe at SOA = 20 msec. (C) Inhibltion by superposition of the two hypothetical response functions at $\mathrm{SOA}=120$ msec.

In the two other parts of Figure 4, two examples of double pulses are presented : summation at SOA $=$ $20 \mathrm{msec}$ and inhibition at SOA $=120 \mathrm{msec}$. The first and second stimuli of the double pulse elicit $f_{1}(t)$ and $f_{2}(t)$, respectively. each of which is the same shape as shown in Part A. Linear superposition of $f_{1}(t)$ and $f_{2}(t)$ is made in the system, and by Assumption 2 the resultant function is expressed as $w_{1} f_{1}(t)+w_{2} f_{2}(t)$, where $w$ represents weighting coefficient. A further assumption for the $w$ coefficient is that (1) $w_{1}=w_{2}$ at very brief SOAs. (2) $w_{1}<w_{2}$ for SOAs at which inhibition occurs, and (3) $w_{1}=w_{2}$ at long SOAs. The shaded parts in $B$ and $C$ are equal to that in $A$. The detection of the double pulse will therefore be at time $\tau_{\text {od }}$ in these figures.

Part B presents a case that is illustrated by setting $w_{1}=w_{2}=1$. The predicted RT will be shorter, because $\tau_{0 d}<\tau_{0}$, as is clear from A and B. Notice herein that the latency time, $\tau$, in the residual stages is supposed to be identical for the single and double pulses. Obviously. Part B is suggestive of summation. Part $C$ shows a tentative case to demonstrate the inhibition. The resultant function is described by setting $w_{1}=0.6$ and $w_{2}=1$. The value of 0.6 is arbitrary. The negative part under $f_{1}(t)$ causes a considerable delay to obtain $\tau_{\text {odd }}$. This will produce longer RT, indicating the inhibition. ${ }^{3}$ The predictions from the above-mentioned cases are clearly consistent with the experimental data.

The $\mathrm{RT}_{0}$ in Equation 1 might be utilized as an estimate of the latency time $\tau$. If $\tau=170 \mathrm{msec}$ as a tentative estimate. then the following approximative RTs will be predicted for the three cases in Figure 4: $21.3 \mathrm{msec}$ for Part A. $196 \mathrm{msec}$ for B. and $307 \mathrm{msec}$ 
for C. These RTs for A and B seem to be adequate, whereas the RT for $C$ is too large in comparison with the present data. This is probably due to the small value of $w_{1}$. To fit the data, therefore, an appropriate value other than 0.6 would be required.

\section{REFERENCES}

Bouman, M. A., \& van den Brink, G. On the integrate capacity in time and space of the human peripheral retina. Journal of the Optical Society of America, 1952, 42. 617-620.

Boynton, R. M. Discrimination of homogeneous double pulses of light. In D. Jameson \& L. M. Hurvich (Eds.). Handbook of senson physiology VII/4: Visual psychophysics. New York: Springer, 1972.

Bruder. G.. \& Kietzman, M. L. Visual temporal integration for threshold, signal detectability, and reaction time measures. Perception \& Psychophysics, 1973, 13, 293-300.

Connors, M. M. Luminance requirements for hue perception and identification, for a range of exposure durations. Joumal of the Optical Society of America, 1970, 60, 958-965.

DAVY. E. The intensity-time relation for multiple flashes of light in the peripheral retina. Joumal of the Optical Society of America. 1952, 42. 937-941.

Grossberg. M. Frequencies and latencies in detecting two-flash stimuli. Perception \& Psychophysics, 1970, 7. 377-380.

HaRTLINE. H. K. Intensity and duration in the excitation of single photoreceptor units. Journal of Cellular and Comparative Physiology, 1934, 5, 229-247.

Hood. D. C.. \& GRover, B. G. Temporal summation of light by a vertebrate visual receptor. Science, 1974, 184, 1003-1005.

IKEDA, M. Temporal summation of positive and negative flashes in the visual system. Journal of the Optical Society of America, 1965, 55, 1527-1534.

Kietzman, M. L., \& Gillam, B. J. Visual temporal integration and simple reaction time. Perception \& Psychophysics. 1972. 11. 333-340.

KELIY, D. H. Visual responses to time-dependent stimuli. II. Single-channel model of the photopic visual system. Journal of the Optical Society of America, 1961, 51, 747-754.

Mansfield, R. J. W. Latency functions in human vision. Vision Research, 1973, 13, 2219-2234.

Pí́ron, H. The sensations. (Trans. M. H. Pirenne \& B. C. Abbot) New Haven: Yale University Press, 1952.
Roufs. J. A. J. Dynamic properties of vision-II. Theoretical relationships between flicker and flash thresholds. Vision Research. 1972, 12, 279-292.

Rours, J. A. J. Dynamic properties of vision-III. Twin flashes, single flashes and flicker fusion. Vision Research, 1973, 13, 309-323.

Sperling. G., \& Sondhi. M. M. Model for visual luminance discrimination and flicker detection. Journal of the Optical Society of America, 1968, 58. 1133-1145.

SPerling, H. G., \& Jolliffe, C. L. Intensity-time relationship at threshold for spectral stimuli in human vision. Journal of the Optical Society of America, 1965, 55, 191-199.

UetsukI. T., \& IKEDA. M. Study of temporal visual response by the summation index. Joumal of the Optical Society of America, 1970, 60, 377-381.

VAN DEN BRINK, G., \& Bouman, M. A. Variation of integrative actions in the retinal system: An adaptational phenomenon. Journal of the Optical Society of America, 1954, 44. 616-620.

VAughan, H. G., JR., Costa, L. D., \& Gilden, L. The functional relation of visual evoked response and reaction time to stimulus intensity. Vision Research, 1966, 6. 645-656.

\section{NOTES}

1. Bruder and Kietzman (1973) have pointed out that the shorter critical duration for RT may be related to the shorter critical duration for neural latency than for frequency or amplitude of the neural responses. From the waveforms of the frog's cone receptor response. Hood and Grover (1974) have predicted that a constant latency as the criterion response would bring a much shorter critical duration than that found when a constant peak response amplitude is used.

2. The equations derived from the models to explain the human temporal frequency characteristics may be available for the theoretical expressions of $\mathrm{ft}$ ) (Kelly. 1961: Roufs. 1972. 1973: Sperling \& Sond hi. 1968).

3. It is to be noted that a possibility to explain the visual backward masking is also involved in the case of $w_{1}<w_{2}$. When $w_{1}=0$ and $\mathrm{n}_{2}>0$. the response function of the first stimulus disappears, and hence the subject is predicted to observe the second stimulus alone.

(Received for publication September 5. 1975; revision accepted November 23, 1975.) 